$\begin{gathered}\text { Revista do Departamento de Geografia } \\ \text { Universidade de São Paulo } \\ \text { www.revistas.usp.br/rdg }\end{gathered}$
Volume Especial - XVII SBGFA / I CNGF (2017)

\title{
Uso de Veículo Aéreo Não Tripulado (Vant) Para Mapeamento das Vulnerabilidades à Inundação Urbana: Referenciais e Bases de Aplicação
}

\author{
Use of Unmanned Aerial Vehicle (Uav) For Mapping of the Vulnerabilities to \\ Urban Flooding: References and Baselines of Application
}

\author{
Elaiz Aparecida Mensch Buffon \\ Universidade Federal do Paraná \\ eambuffon@gmail.com \\ Otacílio Lopes de Souza da Paz \\ Universidade Federal do Paraná \\ otacilio.paz@gmail.com
Tony Vinicius Moreira Sampaio
Universidade Federal do Paraná
tony2sampaio@gmail.com

Resumo: Dados sociais quando integrados aos dados ambientais, possibilitam o mapeamento de vulnerabilidade das áreas que potencialmente podem ser afetadas por inundações. Objetiva-se, neste trabalho, discutir a aplicação de veículo aéreo não-tripulado (VANT) como tecnologia de apoio ao mapeamento das vulnerabilidades em escala de detalhe. A área de estudo foi três setores censitários localizados no município de Campo Magro, Paraná, Brasil. A construção metodológica pautou-se na cartografia de síntese (álgebra de mapas + análise multicritério). Atrelado a essa técnica, elaborou-se o ortomosaico para a área de estudo, a partir de dados coletados com o VANT, possibilitando a desagregação dos setores censitários em unidades de paisagem, que são caracterizadas por elementos que compõem a dimensão do ordenamento urbano. As áreas de preservação permanente (APPs) foram combinadas com o mapeamento de vulnerabilidade social na unidade intra-quadras, para assim, elaborar o mapa composto de vulnerabilidade do lugar a inundação urbana, que permitiu uma melhor aproximação com a realidade.

Palavras chave: Geotecnologias; Veículo Aéreo NãoTripulado; Vulnerabilidades; Unidade de Paisagem; Aplicação.

\begin{abstract}
Social data when integrated with environmental data enable the mapping of vulnerability of areas that could potentially be affected by floods. The objective of this work was to discuss the application of Unmanned Aerial Vehicle (UAV) as assistive technology to the mapping of vulnerabilities in detail. The study area was three census tracts located in the municipality of Campo Magro, Paraná, Brazil. The construction methodology was guided in the cartography of synthesis (maps algebra + multicriteria analysis). Coupled to this technique, we elaborated the ortomosaico to the study area, from data collected with the $\mathrm{UAV}$, allowing the breakdown of census tracts in landscape units, which are characterized by elements that make up the size of urban planning. The permanent preservation areas (PPAs) were combined with the mapping of social vulnerability in intra-blocks, to prepare the composite of vulnerability of the place the urban flooding, which allowed a better approximation to reality.
\end{abstract}

Keywords: Geotechnologies; Unmanned Aerial Vehicle; Vulnerabilities; Unit of Landscape; Application. 


\section{INTRODUÇÃO}

Os dados sociais quando integrados aos dados ambientais permitem mapear as áreas que potencialmente podem ser afetadas por inundações de modo mais eficiente. Assim, é possível apoiar ações que podem ser utilizadas em atividades de gerenciamento de riscos e planejamento frente aos desastres decorrentes de inundações. Essas atividades de acordo com Cardona (1996) são divididas em três momentos, a saber: antes do desastre (prevenção, mitigação, preparação e alerta), durante o desastre (resposta) e, depois do desastre (reabilitação e reconstrução).

Sausen e Narvaes (2015) apontam que existem muitos mapas emergenciais para avaliar inundações, ou seja, aqueles mapas elaborados para a fase de resposta. As mesmas autoras lembram que "há poucos mapas operacionais, com aquisição de imagens de áreas específicas, e muito poucos dados são utilizados para o ciclo de gerenciamento de inundações" (p.121).

$\mathrm{O}$ mapeamento das áreas de risco às inundações depende necessariamente do conhecimento da vulnerabilidade destas. Estudos como Kafle et al. (2006), Rebelo (2010), Grassi et al. (2013) têm buscado realizar mapeamentos de perigo, risco e vulnerabilidade a inundação. Os procedimentos adotados pelas pesquisas têm em comum o uso de Sistemas de Informações Geográficas (SIG) para integrar os processos socioeconômicos e físicos, com o auxílio de modelos de simulações e dados de sensoriamento remoto.

Os dados de sensoriamento remoto precisam serem selecionados de acordo com as características da plataforma/sensor e o objetivo do mapeamento, assim, devem observar a resolução espacial, temporal e espectral, a acessibilidade e o custo dos dados. Um recurso utilizado para obter esses dados, embora ainda muito restrito, em razão do elevado custo têm sido o sensor aerotransportado Light Detection And Ranging (LIDAR), conforme Lim et al. (2003).

Outro recurso que ganha destaque no Brasil, mais recentemente, é o emprego de veículo aéreo nãotripulado (VANT), se apresentando como uma tecnologia de coleta de imagens que subsidiam o mapeamento em grandes escalas (LINHARES et al., 2013). Além dos mapeamentos em grandes escalas, os VANTs possuem custos relativamente baixos e são mais práticos em relação às aeronaves tradicionais, tendo aplicações na agricultura, planejamento urbano, monitoramento e fiscalização de áreas (FERREIRA et al.,2013).

No Brasil, estudos recentes sobre vulnerabilidades aos riscos naturais, principalmente se tratando de inundações e movimentos de massa (MESSIAS e FERREIRA, 2015), apresentam grau elevado de subjetividade, decorrentes tanto da seleção de parâmetros adotados na metodologia, bem como da qualidade dos dados, especialmente, aqueles originários de técnicas de sensoriamento remoto.

Nesse sentido, almeja-se com este trabalho discutir o uso de VANTs como meio para coleta de dados que visam a construção de um mapeamento de vulnerabilidade com alto nível de detalhe. Essa discussão é embasada nos resultados do estudo realizado em três setores censitários de Campo Magro - Paraná, Brasil. A proposta metodológica empregada neste estudo é caracterizada no desenvolvimento do trabalho, bem como a delimitação da área de estudo e dos materiais e métodos utilizados.

\section{METODOLOGIA}

No estudo da vulnerabilidade social e ambiental a inundação urbana, diferentes métodos podem ser empregados para quantificar as características socioeconômicas da população e biofísicas do ambiente, auxiliando, assim no conhecimento dos condicionantes que possam induzir ou colocar em risco a população. Na escolha entre um método ou outro devem ser levadas em consideração fatores como o objetivo do trabalho, a disponibilidade de dados em escala adequada e a viabilidade de execução a partir dos equipamentos disponíveis.

No presente trabalho optou-se por construir um mapeamento integrado da vulnerabilidade social e ambiental a inundação, denominado de vulnerabilidade do lugar. Cutter (1996) descreve que a vulnerabilidade do lugar compreende a vulnerabilidade ambiental (biofísica) que enfoca a probabilidade de exposição, e a social que evidencia a análise da probabilidade de consequências adversas. $\mathrm{O}$ uso de sistemas espaciais, tais como SIGs, permite integrar fontes diversas de dados através de modelagens e construção de indicadores, que, assim, auxiliam na avaliação da vulnerabilidade (CUTTER, 2003). A vulnerabilidade permite expressar a magnitude do 
impacto previsível (inundação) sobre o alvo (população) (VEYRET, 2007), provendo, dessa forma, a base para a gestão de riscos e redução de desastres.

A delimitação da área da coleta de dados em escala de detalhe por meio do VANT foi embasada no mapeamento da vulnerabilidade social, admitindo o aglomerado de setores com maior heterogeneidade de índices de vulnerabilidade social (com condições muito baixo, médio a alto e muito alto) (Figura 1). No estudo em questão, esse aglomerado localiza-se na porção periférica da cidade de Campo Magro - PR.

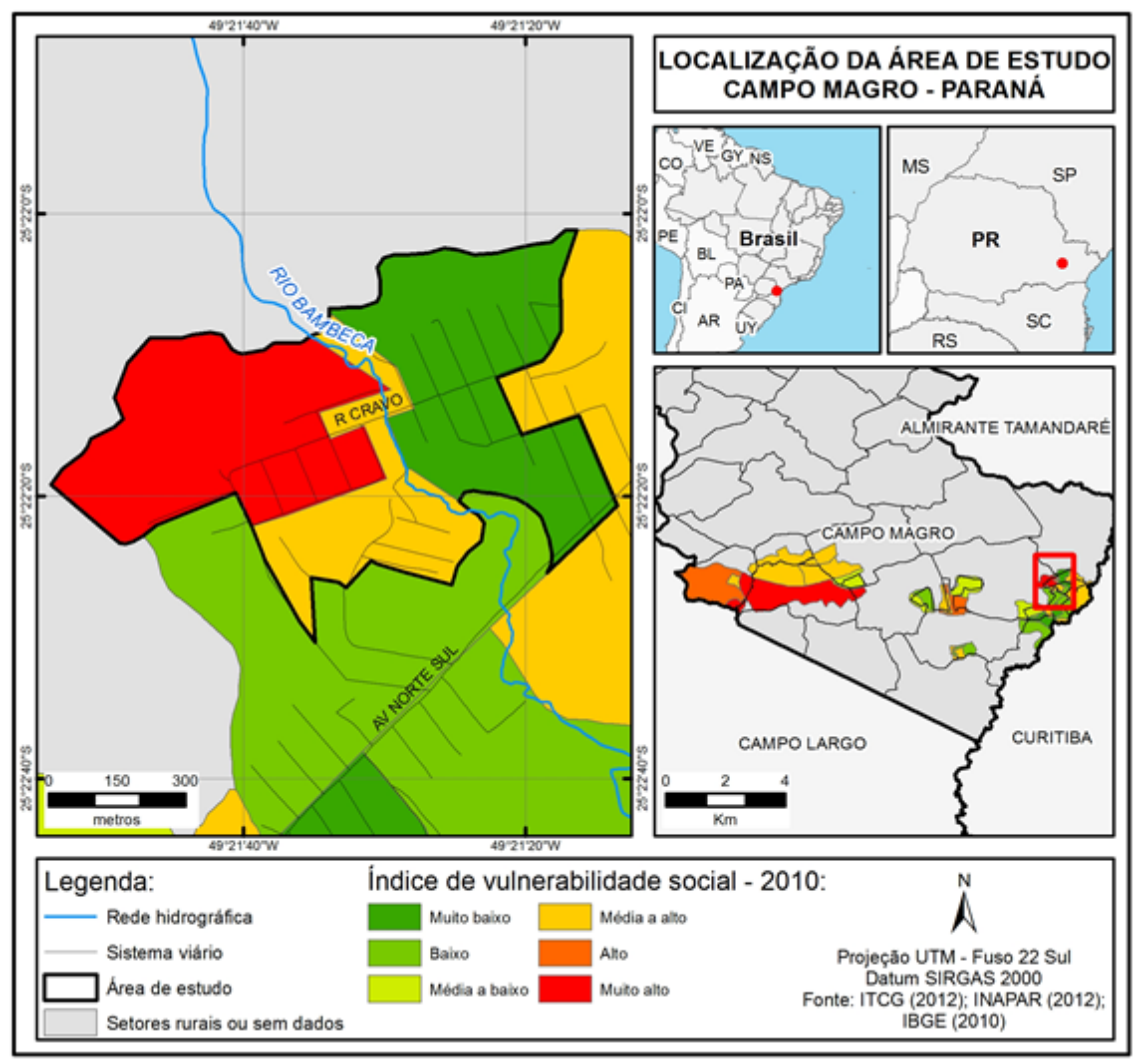

Figura 1: Campo Magro: Delimitação e localização da área de estudo. Elaboração: Autores (2017).

Os dados que foram utilizados no mapeamento da vulnerabilidade social da área de estudo são provenientes do censo de 2010 (IBGE, 2010). A partir desses dados, foi aplicada a técnica de cartografia de síntese (MARTINELLI 1991, 2003; SAMPAIO, 2012), por meio da álgebra de mapas e análise multicritério para expressar a vulnerabilidade social a inundação. Os passos aplicados foram: 1) a normalização dos dados, 2) a padronização dos valores, 3) a eleição e ponderação das variáveis, e 4) o processo de síntese dos valores.

Equação 1:

De acordo com Sampaio (2012) esse processo de síntese em concepção geral é expressado pela

$$
\text { Síntese }=\sum_{i=1}^{n} V_{n p p i}
$$

\section{(Equação 1)}

Onde:

Vnppi - Representa cada uma das variáveis empregadas no processo de síntese, após serem normalizadas, padronizadas e ponderadas, e $i$ varia de 1 (primeira Vnpp) até $n$ (última Vnpp). 
Para o mapeamento da vulnerabilidade social na escala de setores censitários, adotou-se o uso de variáveis relacionadas com a infraestrutura urbana e com as condições socioeconômicas, com pesos distintos (Tabela 1). Essa determinação de variáveis pautou-se na proposta de e Rossini-Penteado e Ferreira (2015).

Tabela 1: Caracterização das variáveis e seus respectivos pesos adotados para o mapeamento da vulnerabilidade social a inundação.

\begin{tabular}{|c|c|c|c|c|}
\hline Categorias & Peso & Subcategoria & Variável (IBGE) & Peso \\
\hline \multirow{3}{*}{ Coleta de esgoto } & \multirow{3}{*}{0,25} & Rede geral & V017 & 0,025 \\
\hline & & Fossa séptica & V018 & 0,075 \\
\hline & & Inadequado & V019, V020, V021, V022 & 0,150 \\
\hline \multirow{3}{*}{$\begin{array}{l}\text { Abastecimento de } \\
\text { água }\end{array}$} & \multirow{3}{*}{0,25} & Rede geral & V012 & 0,025 \\
\hline & & Poço ou nascente & V013 & 0,075 \\
\hline & & Outra forma & V014 e V015 & 0,150 \\
\hline \multirow{3}{*}{ Coleta de lixo } & \multirow{3}{*}{0,25} & Coletado & V035 & 0,025 \\
\hline & & Queimado & V038 & 0,075 \\
\hline & & Inadequado & V039, V040, V041 e V042 & 0,150 \\
\hline \multirow{3}{*}{ Renda } & \multirow{3}{*}{0,25} & 0-1 salários & V01, V02 e V010 & 0,150 \\
\hline & & 2-3 salários & V03 e V04 & 0,075 \\
\hline & & > 3 salários & V05, V06, V07, V08, V09 & 0,025 \\
\hline
\end{tabular}

Elaboração: Autores (2017).

O trabalho de campo para levantamento dos dados geoespaciais (ortoimagem e modelo digital de elevação) foi realizado no dia 06 de fevereiro de 2017. Foi utilizado o VANT modelo Phantom 3 - Professional - DJI, do departamento de Geografia da Universidade Federal do Paraná. Foram realizados 3 voos com duração média de 15 minutos cada.

Para o planejamento do voo foram observadas a variação local do relevo (aproximadamente 30 metros), para fins de evitar colisões com objetos e permitir o ajuste da dimensão média dos pixels (GSD - Ground Sample Distance), a autonomia da aeronave, e as condições meteorológicas (velocidade do vento e umidade). Os parâmetros do voo estão descritos na Figura 2. O aplicativo utilizado para o planejamento da missão foi o pixel4D versão para Android (gratuita).

Os dados obtidos foram processados no software Agisoft PhotoScan, seguindo rotinas para alinhamento e junção das fotografias captadas. Por fim, foi elaborado a ortofoto e o modelo digital de superfície (MDS) da área de estudo, ambos com resolução horizontal de 0,2 metros. Um segundo trabalho de campo foi realizado no dia 09 de fevereiro de 2016, a fim de conhecer a área de estudo, além de levantar pontos para validar a bases cartográficas elaborada a priori. 


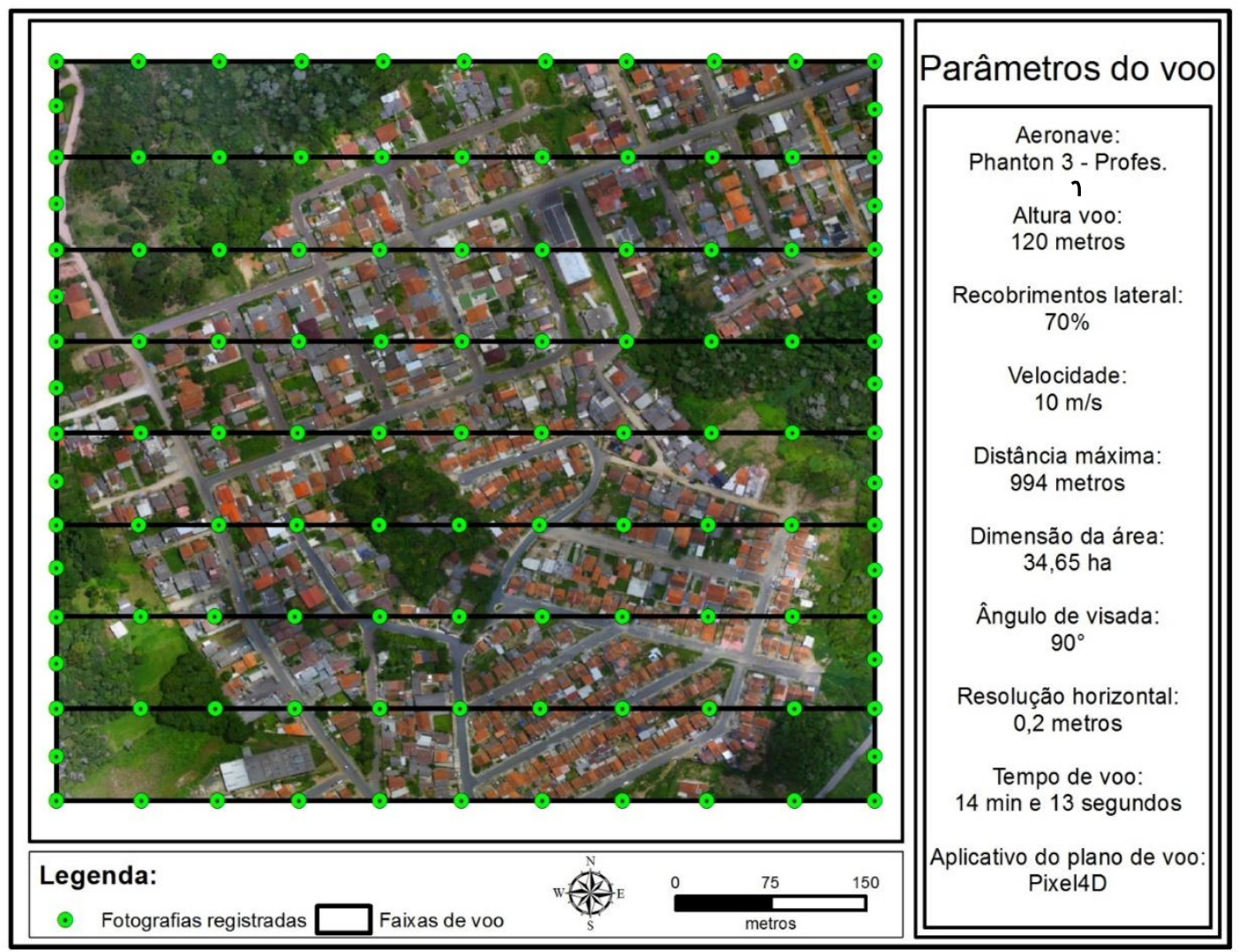

Figura 2: Ilustração de um dos três planos de voo realizado em campo. Elaboração: Autores (2017).

O mapeamento de vulnerabilidade social foi desagregado a partir dos dados levantados com ortofotos para a unidade de quadras. Essa desagregação pautou-se na análise do ordenamento urbano com base nos seguintes elementos que podem interferir no processo de gerenciamento de riscos e respostas frente aos desastres decorrentes de inundações: traçado do sistema viário, pavimentação e vegetação. Esses elementos foram interpretados e integrados na análise de modo a permitir classificar o ordenamento urbano em três classes (Quadro 1). Cabe destacar, que a unidade espacial máxima de interpretação foi o limite de quadras, no entanto, quando as paisagens marcavam uma heterogeneidade dentro da quadra, realizou-se a desagregação por meio de uma nova unidade (intra-quadra) de paisagem homogênea.

Quadro 1: Combinação matricial para obtenção das classes de Ordenamento Urbano utilizado no mapeamento de vulnerabilidade social a inundação na unidade de quadras. Elaboração: Autores (2017) baseado em Rossini-

Penteado e Ferreira (2015).

\begin{tabular}{|c||c||c||c|}
\hline Classe & Traçado do sistema viário & Pavimentação & Vegetação \\
\hline Alto & Sim & Sim & Sim \\
\hline \multirow{3}{*}{ Médio } & Sim & Sim & Não \\
\cline { 2 - 4 } & Sim & Não & Sim \\
\cline { 2 - 4 } & Não & Sim & Sim \\
\hline \multirow{3}{*}{ Baixo } & Não & Não & Não \\
\cline { 2 - 4 } & Não & Sim & Não \\
\cline { 2 - 4 } & Sim & Não & Não \\
\cline { 2 - 4 } & Não & Não \\
\hline
\end{tabular}


Essas classes do ordenamento urbano foram combinadas com os índices de vulnerabilidade social obtidos na unidade de setores. No Quadro 2 é possível visualizar a caracterização das combinações que geraram o índice final de vulnerabilidade social na unidade máxima da desagregação em quadras.

Quadro 2: Combinação matricial das classes de ordenamento urbano com os índices de vulnerabilidade social (setores) para obtenção do índice de vulnerabilidade social (quadras). Elaboração: Autores (2017).

\begin{tabular}{|c||c|c|c||}
\hline \multirow{2}{*}{\multicolumn{1}{|c|}{ IVS }} & \multicolumn{3}{c|}{ Classes do Ordenamento } \\
Urbano \\
\cline { 2 - 4 } & Alto & Médio & Baixo \\
\hline \hline Muito Baixo (MB) & $\mathrm{MB}$ & $\mathrm{MB}$ & $\mathrm{B}$ \\
\hline Baixo (B) & $\mathrm{MB}$ & $\mathrm{B}$ & $\mathrm{MaB}$ \\
\hline $\begin{array}{c}\text { Médio a Baixo } \\
\text { (MaB) }\end{array}$ & $\mathrm{B}$ & $\mathrm{MaB}$ & $\mathrm{MaA}$ \\
\hline \hline Médio a Alto (MaA) & $\mathrm{MaB}$ & $\mathrm{MaA}$ & $\mathrm{A}$ \\
\hline \hline Alto (A) & $\mathrm{MaA}$ & $\mathrm{A}$ & $\mathrm{MA}$ \\
\hline Muito Alto (MA) & $\mathrm{A}$ & $\mathrm{MA}$ & $\mathrm{MA}$ \\
\hline
\end{tabular}

Sendo a área de estudo urbana, aplicou-se a concepção de Dauphiné (2001), que não considera exclusivamente o caráter matemático ligado a probabilidade de ocorrência de um risco, mas enfatiza o uso da função e relação em um caráter multicausal. Nesse sentido, foram delimitadas as áreas de preservação permanente (APPs) de todos os rios que percorrem a área, independente de no momento atual estarem retificados, canalizados e aterrados. As APPs dos rios foram definidas com base na interpretação do novo Código Florestal Brasileiro (BRASIL, 2012), sendo demarcado uma faixa de 30 metros no entorno do respectivo corpo hídrico. A matriz de combinação entre os índices de vulnerabilidade social (quadras) e as APPs é apresentada no quadro 3. É importante enfatizar, que essa combinação se pautou na relação entre as áreas, tendo como base a ocupação de APPs.

Quadro 3: Combinação matricial dos índices de vulnerabilidade social (quadras) com a vulnerabilidade ambiental para obtenção dos índices de vulnerabilidade do lugar. Elaboração: Autores (2017).

\begin{tabular}{|c|c|c|c|}
\hline \multirow{2}{*}{ IVS } & \multicolumn{3}{|c|}{$\begin{array}{l}\text { APPs em áreas edificadas (porcentagem da } \\
\text { área) }\end{array}$} \\
\hline & Até $50 \%$ & $\begin{array}{l}\text { Mais de } \\
50 \%\end{array}$ & $0 \%$ \\
\hline Muito Baixo (MB) & B & $\mathrm{MaB}$ & $\mathrm{MB}$ \\
\hline Baixo $(\mathrm{B})$ & $\mathrm{MaB}$ & $\mathrm{MaA}$ & $\mathrm{MB}$ \\
\hline Médio a Baixo (MaB) & $\mathrm{MaA}$ & $\mathrm{A}$ & B \\
\hline Médio a Alto (MaA) & $\mathrm{A}$ & $\overline{\mathrm{MA}}$ & $\mathrm{MaB}$ \\
\hline Alto $(\mathrm{A})$ & $\mathrm{MA}$ & $\mathrm{MA}$ & $\mathrm{MaA}$ \\
\hline Muito Alto (MA) & $\mathrm{MA}$ & $\mathrm{MA}$ & $\mathrm{A}$ \\
\hline
\end{tabular}

A importância vinculada com a opção dessas combinações consiste em expressar com maior nível de detalhe as condições de vulnerabilidades. Longley et al., $(2013$, p.92) afirmam que "o nível de detalhe de uma base de dados em SIG, é uma das suas propriedades mais importantes, já que determina tanto o grau de aproximação desta base com o mundo real como a sua complexidade". 


\section{RESULTADOS E DISCUSSÃO}

Nesse item, em um primeiro momento, é apresentado o mosaico das ortofotos geradas a partir do levantamento de campo, e que foram utilizadas para a interpretação das informações que permitiram a construção dos dados de ordenamento urbano. Em conjunto com esse mosaico, apresenta-se fotos levantadas em campo que permitem caracterizar pontos chaves para determinação da base hidrográfica da área de estudo.

Analisando o ortomosaico da área de estudo (Figura 3) observa-se que a área total de estudo representa $441.225,05 \mathrm{~m}^{2}$, sendo que $33,72 \%$ desta corresponde a vegetação densa, 0,65\% é ocupada por corpos hídricos (lagos) e, 65,62\% equivale as áreas edificadas.

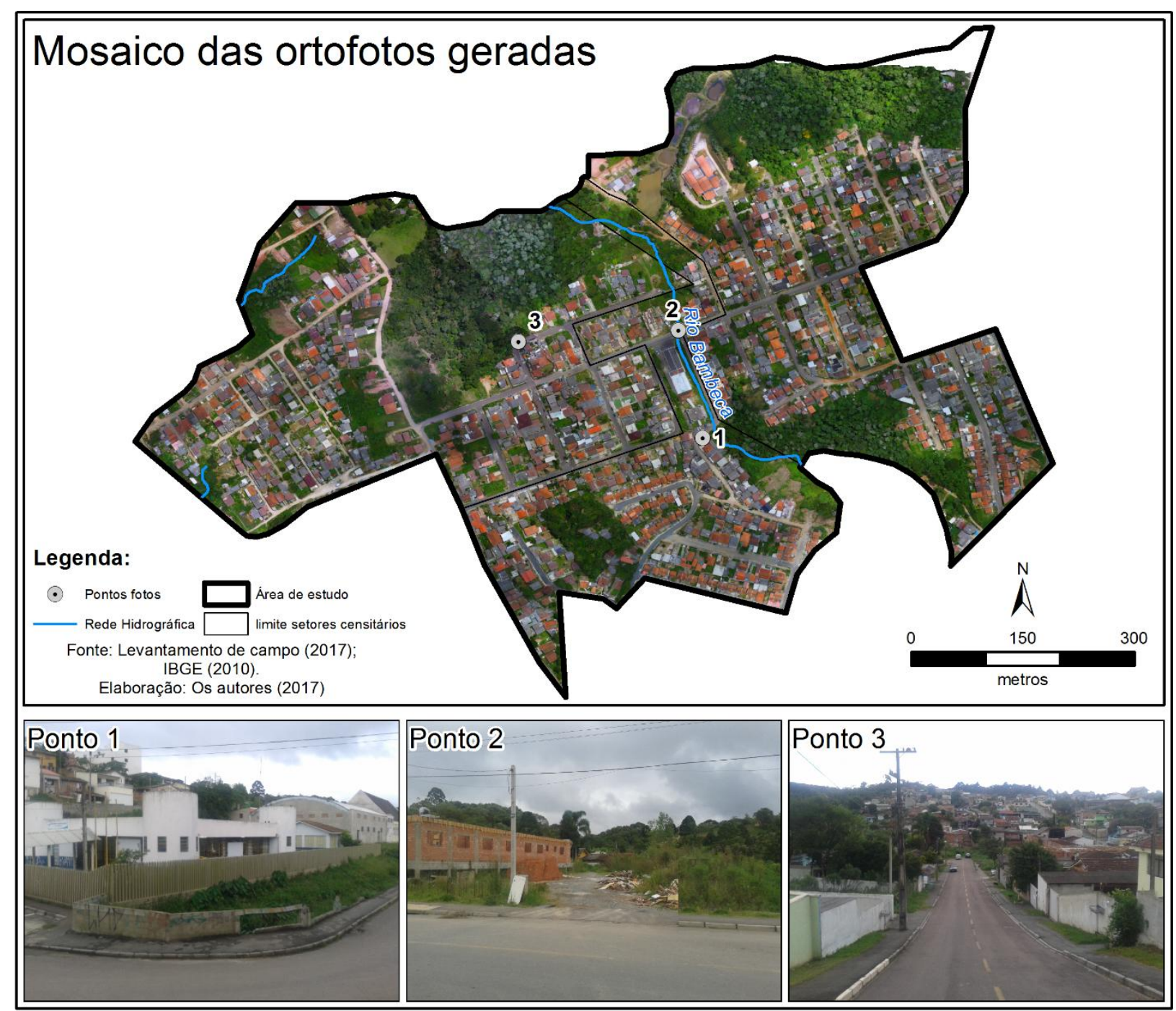

Figura 3: Campo Magro: mosaico das ortofotos, representação da hidrografia e registros fotográficos da área. Fotos e elaboração: Os autores (2017).

A interpretação das ortofotos permitiu realizar análises específicas sobre a paisagem, como por exemplo, na dimensão dos elementos que compreende a abordagem do ordenamento urbano. Observou-se que $61,31 \%$ das estradas são pavimentadas e 38,69\% não pavimentadas. As classes de ordenamento urbano nas áreas edificadas foram assim caracterizadas: 17,38\% com alto índice de ordenamento, e 45,80\% com as condições de médio índice de ordenamento, e por fim, 36,82\% da área com baixo índice de ordenamento. 
A Figura 4 permite visualizar o delineamento dos índices de vulnerabilidade do lugar a partir das combinações de atributos socioeconômicos e físicos, tendo como limite máximo a unidade de quadras.

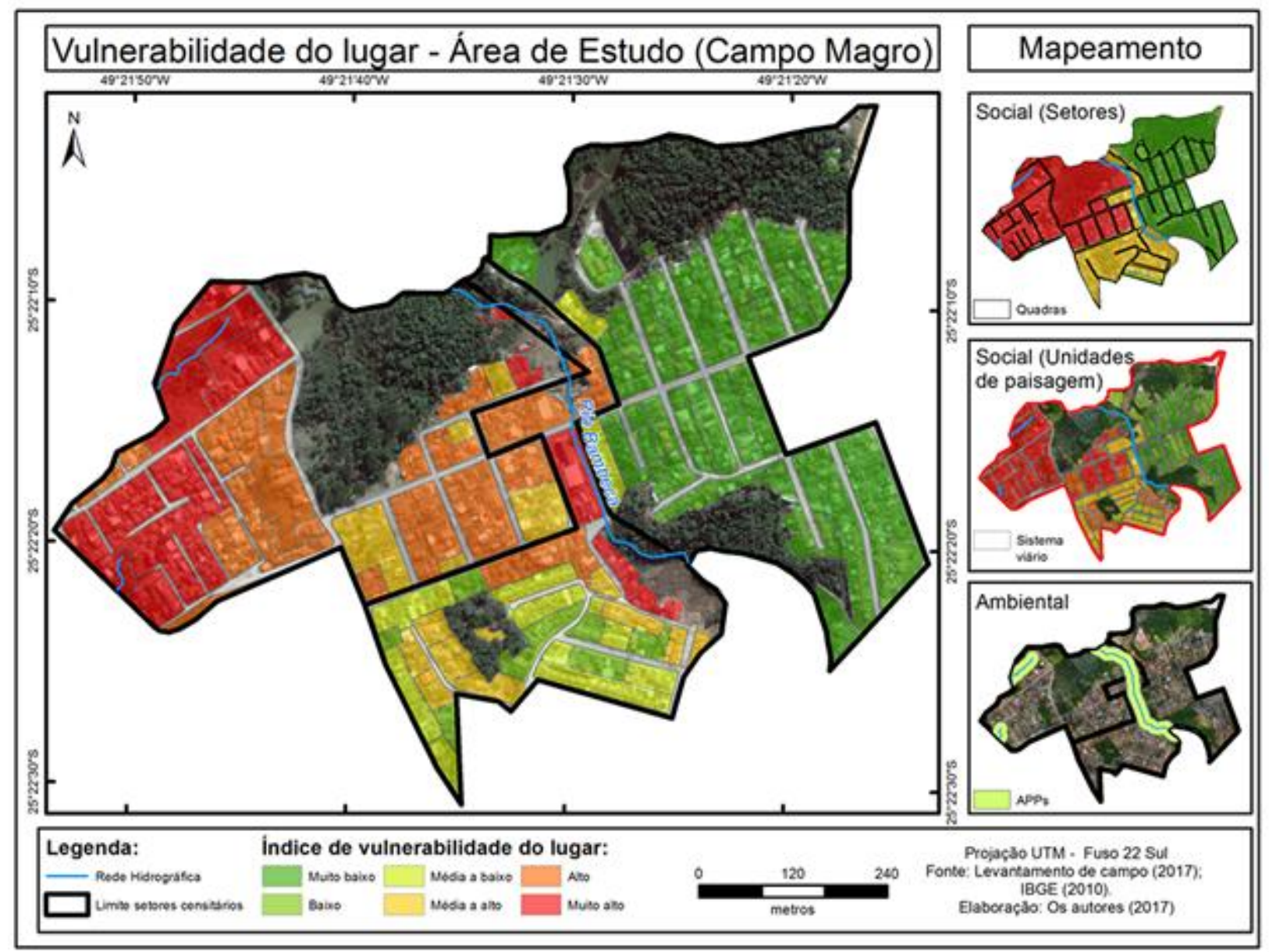

Figura 4: Campo Magro: mapeamentos que combinados formam o mapa composto com o índice de vulnerabilidade do lugar a inundação. Elaboração: Os autores (2017).

Os índices de vulnerabilidade social foram desagregados em 3 setores e assim caracterizados como muito alto, com 39,39\% da área total, médio a alto, 21,36\% e, muito baixo correspondendo a 39,24\% da área de estudo.

O segundo mapeamento de vulnerabilidade social, resultante do cruzamento das classes de ordenamento urbano com a vulnerabilidade social por unidade de setores foi delineado a partir de seis índices na unidade máxima de desagregação de quadras, sendo que no total foram definidas 116 unidades de paisagem para análise das áreas edificadas. A caracterização dessas unidades é representada por índices obtidos a partir do segundo mapeamento de vulnerabilidade social, da seguinte forma: muito baixo $(26,92 \%)$; baixo $(8,19 \%)$; médio a baixo $(4,13 \%)$; médio a alto $(14,43 \%)$; alto $(10,40 \%)$; muito alto $(35,94 \%)$.

No que se refere ao mapeamento com a dimensão ambiental, depreende-se que 9,98\% da área de estudo está inserida em APPs, compreendendo uma área de $44.035,28 \mathrm{~m}^{2}$. Considerando esses atributos socioeconômicos e físicos foi possível desagregar em níveis de maior detalhe a representação das condições de vulnerabilidade, resultando, assim, em um mapa composto que representa a condição de vulnerabilidade do lugar à inundação.

Em linhas gerais, os índices de vulnerabilidade do lugar a inundação ficam assim caracterizados em termos percentuais quando comparados com a área total edificada: muito baixo (33,46\%), baixo (4,13\%), médio a baixo $(9,31 \%)$, médio a alto $(9,40 \%)$, alto $(23,46 \%)$ e muito alto $(20,23 \%)$. 
A comparação entre os mapeamentos de vulnerabilidades com o mapa composto de vulnerabilidade do lugar permite perceber que esse último se trata de uma representação espacial com maior aproximação da realidade, quando comparado aos demais. Nesse contexto, entende-se que o uso do VANT para levantamento de dados geoespaciais é de extrema importância no mapeamento de vulnerabilidades, uma vez que esse permite avaliar dados secundários obtidos, e auxiliar na redução do grau de incerteza sobre as características do local de estudo.

Longley et al., (2013, p.166) chamam atenção para o fato de que "boas análises em SIG não podem substituir concepções ou representações geográficas pobres - elas podem sinalizar as prováveis consequências de ambas". Atentando-se para isso, não se pretende com o presente trabalho invalidar o mapeamento de vulnerabilidade social na unidade de setores censitários, mas justamente, evidenciar a incerteza na análise de fenômenos geográficos, e levantar uma proposta metodológica que visa uma escala ou nível de detalhamento geográfico mais refinado no conhecimento das vulnerabilidades.

\section{CONCLUSÕES}

Os procedimentos implementados, referentes ao processo de cruzamentos de mapas, elaboração de mosaico e interpretação da ortofoto, e delimitação das APPs, mostraram-se de relevante importância para melhor o nível de detalhamento dos resultados. O uso do SIG foi fundamental para sintetizar um grande número de dados, ao mesmo tempo em que permitiu atualizar a base de dados disponível.

As análises estatísticas, realizadas para compreender os delineamentos dos índices de vulnerabilidades em cada um dos mapeamentos, apresentaram variações quando comparadas. Essas variações entre os mapeamentos permitem expressar os avanços da representação frente ao objetivo da aproximação da realidade no contexto espaço-temporal.

Constatou-se, ainda, que as maiores variações ocorreram nos índices de muito alto e alto, o que implicou em um melhor detalhamento dessas condições na área de estudo. Nesse contexto, a área com menor alteração dos índices foi o setor que no mapeamento de vulnerabilidade social por setores estava caracterizado pelo índice de muito baixo. Esse aspecto permite inferir a importância de determinar zonas prioritárias, aquelas de alta e muito alta vulnerabilidade, para mapeamentos que visem maiores níveis de detalhes.

Assim, os resultados aqui encontrados e discutidos permitem concluir que o VANT se mostrou eficaz para auxiliar o aumento no nível de aproximação da realidade. Considera-se, todavia, a realização de novos estudos que demonstrem a validade dos índices de vulnerabilidades frente às ocorrências de inundações. Além disso, é importante a continuidade de estudos com a aplicação do VANT, a fim de, demonstrar a simplicidade e o baixo custo atrelado a essa ferramenta e sua relevância para pesquisas socioambientais.

\section{REFERÊNCIAS}

BRASIL. Lei Federal no 12.727, de 17 de outubro de 2012. Diário Oficial [da] da República Federativa do Brasil. Brasília: Congresso Nacional, 2012.

CARDONA, O. D. El manejo de riesgos y los preparativos para desastres: compromiso institucional para mejorar la calidad de vida. In: MASKREY, A. Desastres: modelo para armar. Colección de piezas de um rompecabezas social. Capítulo 9 - 1996.

CUTTER, S. L. Vulnerability to environmental hazard. Progress in Human Geography, v. 20, n.4, 1996, p.529-539.

CUTTER, S. L. The vulnerability of science and the science of vulnerability. Annals of the Association of American Geographers, p. 1-12, 2003.

DAUPHINÉ, A. Risques et catastrophes - Observer, spatialiser, comprendre, gerer. Paris: Armand Colin, 2001. 
FERREIRA, A. M. R.; ROIG, H. L.; MAROTTA, G. S.; MENEZES, P. H. B. J. Utilização de aeronaves remotamente pilotadas para extração de mosaico georreferenciado multiespectral e modelo digital de elevação de altíssima resolução espacial. In: Anais do Simpósio Brasileiro de Sensoriamento Remoto (SBSR) INPE, 16., 2013, Natal, 2013, p. 9308-9315.

GRASSI, J.; DEPPE, F.; LOHMANN, M.; SANGOI, D. S. Mapeamento da vulnerabilidade a inundações e deslizamentos de terra no estado do Paraná. In: Anais do Simpósio Brasileiro de Sensoriamento Remoto, SBSR - INPE, 16., Foz do Iguaçu, 2013, p.2051-2058.

IBGE. Base de informações do Censo Demográfico 2010: resultados do universo agregados por setor censitário. Rio de Janeiro, 2011b.

KAFLE, T. P.; HAZARIKA, M. K.; SHRESTHA, K. C.; PRATHUMCHAI, K.; SAMARAKOOM, L. Integration of remote sensing and GIS model with flood simulation model for flood hazard mapping in the Bagmati river, Nepal. In: International Symposium on New Technologies for Urban Safety of Megacities in Asia, 5., Phuket Thailand, 2006, p. 257-268.

LIM, K.; TREITZ, P.; WULDER, M.; ST-ONGE, B.; FLOOD, M. LiDAR remote sensing of forest structure. Progress in Physical Geography, v. 27, n. 1, p. 88-106, 2003.

LINHARES, M. M. A.; ROCHA, N. C. C.; AMARAL, B. A. S. Análise do índice MPRI como indicador vegetativo através da correlação do mesmo com o índice NDVI. In: Anais do Simpósio Brasileiro de Sensoriamento Remoto, SBSR - INPE, 16., Foz do Iguaçu, 2013. p. 8254-8260.

LONGLEY, P. A; GOODCHILD, M. F.; MAGUIRE, D. J; RHIND, D. W. Sistemas e ciência da informação geográfica. 3. Ed., Porto Alegre: Bookman, 2013, 540 p.

MARTINELLI, M. Curso de cartografia temática. 1. ed. São Paulo: Contexto, 1991. Mapas da geografia e cartografia temática. São Paulo: Contexto, 2003.

MESSIAS, C. G.; FERREIRA, M. F. M. Densidade de processos erosivos e fragilidade ambiental em sub-bacias hidrográficas ao sul do reservatório do Funil, Alto Rio Grande (MG). In: FREITAS, M. I. C. de.; LOMBARDO, M. A.; ZACHARIAS, A. A. Vulnerabilidades e riscos: reflexões e aplicações na análise do território. Rio Claro (SP): UNESP - IGCE-CEAPLA, 2015, p.55-62.

REBELO, F. Geografia Física e Riscos Naturais. Coimbra: Imprensa da Universidade de Coimbra, 2010.

ROSSINI-PENTEADO, D.; FERREIRA, C. J. Mapeamento da vulnerabilidade para análise de riscos associados a processos geodinâmicos. In: FREITAS, M. I. C. de.; LOMBARDO, M. A.; ZACHARIAS, A. A. Vulnerabilidades e riscos: reflexões e aplicações na análise do território. Rio Claro (SP): UNESP - IGCECEAPLA, 2015, p.55-62.

SAMPAIO, T. V. M. Diretrizes e procedimentos metodológicos para a cartografia de síntese com atributos quantitativos via álgebra de mapas e análise multicritério. Boletim de Geografia, Maringá, v. 30, n. 1, p. 121131, 2012.

SAUSEN, T. M. e NARVAES, I. da S. Sensoriamento remoto para inundação e enxurrada. In: SAUSEN, T. M. \& LACRUZ, M. S. P. Sensoriamento Remoto para desastres. São Paulo: Oficina de Textos, 2015, p. 119147.

VEYRET, Y \& RICHEMOND, N. M. Os tipos de riscos. In: Veyret, Y. Os riscos: O homem como agressor e vítima do meio ambiente. São Paulo: Contexto, 2007. p.63-76. 